\title{
LASER TRAPPING BASED ON PHOTOPHORETIC FORCES USING A SPATIAL LIGHT MODULATOR
}

\author{
A.P. Porfirev, A.S. Shipilov \\ Samara National Research University, Samara Russia \\ Image Processing Systems Institute - Branch of the Federal Scientific Research Centre "Crys- \\ tallography and Photonics" of Russian Academy of Sciences
}

\begin{abstract}
We demonstrate laser manipulation of light-absorbing particles in air using a spatial light modulator. By controlling the three-dimensional distribution of the generated optical field, we demonstrate how to control the position of individual trapped particles dynamically and independently. The transfer of a trapped particle from one optical trap to another is shown.
\end{abstract}

Keywords: laser trapping, photophoresis, photophoretic forces, spatial light modulator, light-absorbing particles.

Citation: Porfirev AP, Shipilov AS. Laser trapping based on photophoretic forces using a spatial light modulator. CEUR Workshop Proceedings, 2016; 1638: 111-116. DOI: 10.18287/1613-0073-2016-1638-111-116

\section{Introduction}

Various primary biological aerosol particles, which include bacteria, fungal spores, plant pollens, small fragments of plants or fungi, and secondary biological aerosol particles, such as the particles formed by an ozone-initiated polymerization of terpenes are potential carriers of a number of human or plants diseases (e.g., inhalation anthrax) [1-3]. Therefore there is a need to study their behavior and properties.

Currently, for the purpose of detection and analysis of biological aerosols various optical methods, including microscopy and spectroscopy, are used [4-5]. Most of these techniques have been developed for particles in air samples which passed through the measuring device. There is a need for devices which could be used to analyze individual aerosols in real time. In such cases, Raman spectroscopy would be more informative. The key problem in the spectroscopy measurement for air-borne particles is that there is a requirement to keep an analyzed particle stationary during measurement. Therefore, methods in which the air sample is passed through a measuring device are not suitable.

In order to solve the problem of retaining particles, optical tweezers based on photophoretic forces can be used [6-8]. Photophoretic forces are several orders of magnitude greater than radiation pressure, so low-power lasers can be used to trap lightabsorbing particles in gaseous media $[9,10]$. The holographic optical tweezers tech- 
nique using spatial light modulators enables simultaneous parallel manipulation of a variety of micro-objects [11]. The optical tweezers technique permits movement of the trapped objects in order to measure and dynamically carry out the change of the samples.

Thus, the combination of a spatial light modulator (for generation of multiple light traps) and a spectroscopic measurement device (for spectral measurements) allows parallel analysis of many aerosols trapped in the test volume. Using low-power lasers and compact hyperspectral cameras [12-14], allows the creation of a compact device, which will enable the analysis of samples in real time.

\section{Design method}

In the case of a positive photophoresis, light-absorbing air-borne particles move away from the light source, and in the case of negative photophoresis - move towards the light source [15]. Therefore, until recently it was thought impossible to carry out three-dimensional laser trapping of light-absorbing particles in air with a single beam, with a Gaussian intensity distribution. In [8], by means of theoretical explanation, authors have shown that stable three-dimensional laser trapping becomes possible if the trapped particles have a non-spherical shape.

Laser trapping the light-absorbing particles in the air with a Hermite-Gaussian TEM10 laser beam has been demonstrated previously [16]. In this case, two light peaks were generated in the focal plane of a focusing micro-objective. In each of these peaks, we were able to capture a single particle.

The "superposition method prisms and lenses," technique can be used to generate an array of light traps [17]. In this case, the transmission function of the diffractive optical element (DOE) generating $\mathrm{N}$ light traps is the following:

$T(x, y)=\sum_{n=1}^{N} \exp \left(-\frac{k\left[x^{2}+y^{2}\right]}{2 f_{n}}\right) \exp \left(i\left[x \omega_{x n}+y \omega_{y n}\right]\right)$,

where $k=2 \pi / \lambda$ is the wavenumber, $f_{n}$ is a distance where $n$th trap is formed, $\omega_{x n}=\frac{2 \pi}{\lambda} \Delta \varepsilon$, and $\omega_{y n}=\frac{2 \pi}{\lambda} \Delta \eta$, are spatial frequencies of $n$th light trap (see Fig. 1).

As shown above, the transmission function of the element is complex. In the case of a small number of generated light traps (for example, $N=\overline{1 . .10}$ ), we can ignore the amplitude of the transmission function and use a single phase-only element. With a large number of generated traps, various methods of amplitude encoding can be used to obtain the single phase-only element $[18,19]$. Our research has shown that the best method of forming the array of light traps is achieved by using a pseudo-random modulation algorithm optimized for a spatial light modulator (SLM) [20]. 


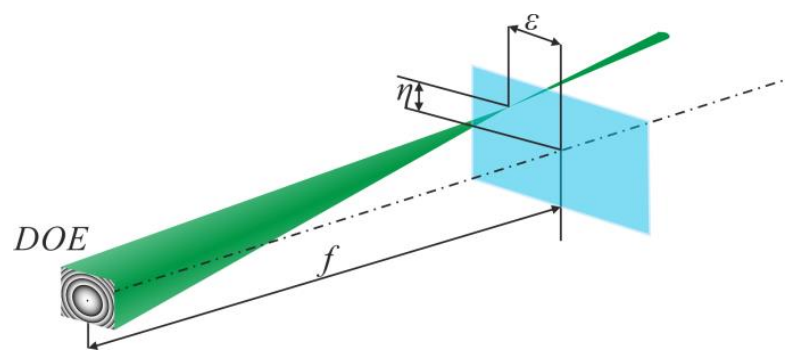

Fig. 1. Generating the optical traps with help "superposition method prisms and lenses"

\section{Experimental investigation of laser trapping using a spatial light modulator}

In order to generate an arbitrary configuration of light traps, a spatial light modulator PLUTO VIS was utilised. The experimental optical setup is shown in Fig. 2. The output laser beam of solid-state laser $(\lambda=532 \mathrm{~nm}$, with a maximum output power of $1500 \mathrm{~mW})$ was expanded with a telescope composed of a micro-objective, $M O_{I}(8 \times$, $\mathrm{NA}=0.2)$, and a lens, $L_{l}\left(f_{1}=350 \mathrm{~mm}\right)$ to illuminate the SLM. Then, lenses $L_{2}$ and $L_{3}$ with focal lengths $f_{2}=350 \mathrm{~mm}$ and $f_{3}=150 \mathrm{~mm}$, respectively, formed an image of the plane conjugated to the SLM display in the focal plane of the micro-objective $\mathrm{MO}_{2}$ $(16 \times, \mathrm{NA}=0.3)$ An airborne light-absorbing particle in a cuvette $C$ was trapped in the area of the focal point of a micro-objective. Observation of the particle trapping was possible due to the scattered light recorded by the video camera, Cam (TOUPCAM UHCCD00800KPA, $1024 \times 768$ pixels). The particles were imaged through a microobjective, $\mathrm{MO}_{3}(8 \times, \mathrm{NA}=0.2)$.

In the experiments, the agglomeration of carbon particles was studied, the transverse dimensions of which were in a range from a few to tens of micrometers. These particles have a non-spherical shape, and therefore ideally suited for experiments of this kind that has been shown in previous experiments [10, 16, 21].

Figure 3 shows the experimental results of how the position of each of the trapped particles using a spatial light modulator can be controlled dynamically. Changing the phase transmission function calculated in accordance with Eq. (1), we can change the position of the formed intensity peaks independently of each other. Thus, in these experiments, we move one of the formed three light traps along the $z$ axis. The particles trapped in the vicinity of the trap move behind them. The velocity the trapped particle is $1.8 \pm 0.1 \mu \mathrm{m} / \mathrm{s}$. Thus, SLMs may be used to dynamically control the position of trapped air-borne light-absorbing micro-particles, which is analogous to the optical manipulation of dielectric micro-objects in a liquid [10]. The difference is only in the trapping mechanism: (1) in the case of dielectric particles in the liquid, gradient force and scattering force play a key role, (2) in the case of light-absorbing particles in the air, photophoretic forces dominate. 


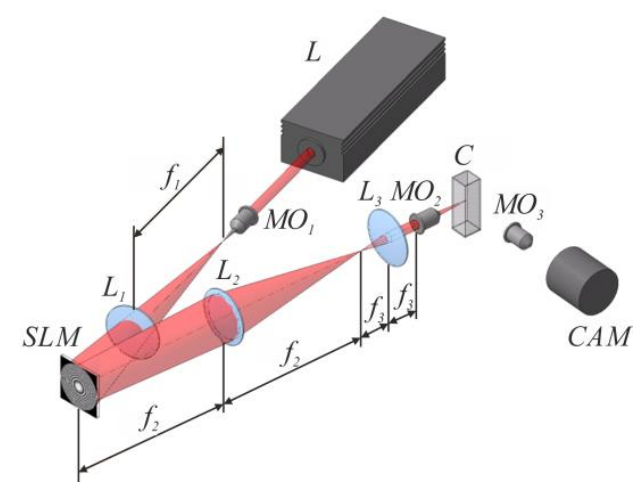

Fig. 2. Experimental optical setup: $L$ is a solid-state laser, $M O_{1}$ is a micro-objective ( $8 \times$, $\mathrm{NA}=0.2), M \mathrm{O}_{2}$ is a micro-objective $(8 \times, \mathrm{NA}=0.2), L_{1}, L_{2}$, and $L_{3}$ are the lenses with focal lengths $f_{1}=250 \mathrm{~mm}, f_{2}=350 \mathrm{~mm}$, and $f_{3}=150 \mathrm{~mm}$, respectively, $M O_{2}$ is a microobjective $(16 \times, \mathrm{NA}=0.3), \mathrm{SLM}$ is a spatial light modulator (PLUTO Spatial Light Modulator, $1920 \times$ 1080 pixel resolution), $C A M$ is a video camera (LOMO TC-1000), $C$ is a cuvette with air-borne light-absorbing particles

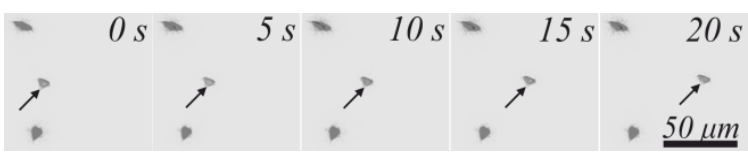

Fig. 3. Experiment on moving particles trapped in the various traps, independently of one another. Black arrow marked the moving particles

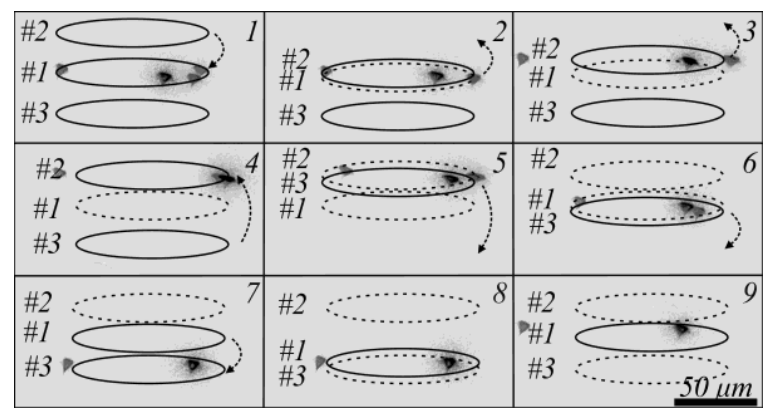

Fig. 4. Sequential transfer the trapped particles from one light trap to another light trap. Dotted outlines indicate "offline trap" solid contours - "online trap", the dotted arrow indicates the direction of movement of the corresponding traps

Figure 4 shows the motion of particles that are consistently transferred from one generated trap to another, and then to a third generated trap. To do this, we generated three light traps and one of them held particles (trap \#1). The trap \#2 was generated next to the trap \#1 (see Fig. 4, frame 2). Then we "turned" the trap \#1 "off". After that we "turned" trap \#1 "off", particles were captured in the trap \#2. After that, we moved the particle using the light trap \#2. Similar operations were carried out to transfer 
particles from the light trap \#2 into the light trap \#3 (see Fig. 4, frames 4-6) and then to transfer particles from the light trap \#3 into the light trap \#1 (see Fig. 4, frames 7 9).

Figure 5 shows the motion of particles trapped by a generated light trap. In this experiment, we generated two light traps (\#1 and \#2 in Fig. 5). The distance between these two light traps is about $30 \mu \mathrm{m}$. First, the particle was trapped by light trap \#1. At that moment, we started to decrease the energy used for the generating the light trap \#1. At the same time, energy used in generating light trap \#2 was not changed. When the energy of light trap \#1 became equal to $50 \%$ of the energy of light trap \#2, the trapped particle left trap \#1 and was trapped in the light trap \#2. With decreasing energy of the generated laser trap, photophoretic forces also decrease. After some time, gravity acting on the trapped particle becomes greater than the photophoretic forces, and the particle leaves the trapping region. The dimensions of trapping region of the generated traps are generally determined by the depth of focus [22]. Based on the results presented in Ref. [22] we can conclude that in this experiment the longitudinal dimension of the trapping region is about $450 \mu \mathrm{m}$. As mentioned above, the distance between the generated light traps is about $30 \mu \mathrm{m}$ (along the propagation axis of the beam). Therefore, when the trapped particle leaves trap \#1, it does not settle at the bottom of the cuvette, and enters into the trapping region of the trap \#2 and is retained there. Then we relocated the trap\#2 down at a velocity of about $2.7 \pm 0.1 \mu \mathrm{m} / \mathrm{s}$.

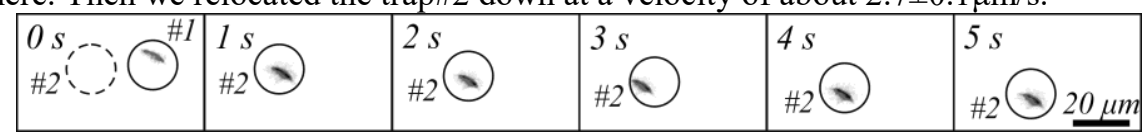

Fig. 5. - Sequential transfer the trapped particles from one light trap to another light trap. The distance between the generated light traps is about $30 \mu \mathrm{m}$

\section{Conclusion}

The paper demonstrates the potential for dynamic control of light-absorbing particles using a spatial light modulator. Using a light modulator made it possible to control the position of the independent, individual traps formed, which increased flexibility in manipulating the process. In addition, we demonstrated the potential of transfering trapped particles from one trap to another by either "turning" one of the traps "off" or by reducing trap energy.

\section{References}

1. Wang Ch, Pan Y-L, Hill SC, Redding B. Photophoretic trapping-Raman spectroscopy for single pollens and fungal spores trapped in air. Journal of Quantitative Spectroscopy and Radiative Transfer, 2015; 153: 4-12.

2. Pöschl U. Atmospheric aerosols: composition, transformation, climate and health effects. Angewandte Chemie - International Edition, 2005; 44(46): 7520-7540.

3. Ramanathan V, Crutzen PJ, Kiehl JT, Rosenfeld D. Atmosphere: Aerosols, climate, and the hydrological cycle, 2001; 294(5549): 2119-2124. 
4. Weber RW. Polen identification. Annals of Allergy, Asthma and Immunology, 1998; 80: 141-147.

5. Tripathi A, Jabbour RE. Bioaerosol analysis with Raman chemical imaging microspectroscopy. Analytical Chemistry, 2009; 81: 6981-6990.

6. Desyatnikov AS, Shvedov VG, Rode AV, Krolikowski W, Kivshar YuS. Photophoretic manipulation of absorbing aerosol particles with vortex beams: theory versus experiment. Optics Express, 2009; 17(10): 8201-8211.

7. Pan Y-L, Wang C, Hill SC, Coleman M, Bereshev LA, Santarpia JL. Trapping of individual airborne absorbing particles using a counterflow nozzle and photophoretic trap for continuous sampling and analysis. Applied Physics Letters, 2014; 104(11): 113507.

8. Zhang Z, Cannan D, Liu J, Zhang P, Christodoulides DN, Chen Z. Observation of trapping and transporting airborne absorbing particles with a single optical beam. Optics Express, 2012; 20(15): 16212-16217.

9. Shvedov VG, Desyatnikov AS, Rode AV, Krolikowski W, Kivshar YuS. Optical guiding of absorbing nanoclusters in air. Optics Express, 2009; 17(7): 5743-5757.

10. Porfirev AP, Skidanov RV. Manipulation of light-absorbing particles in air with optical bottle arrays. Computer Optics, 2014; 38(4): 722-726.

11. Grier DG. A revolution in optical manipulation. Nature, 2003; 424(6950): 810-816.

12. Kazanskiy NL, Kharitonov SI, Doskolovich LL, Pavelyev AV. Modeling the performance of a spaceborne hyperspectrometer based on the Offner scheme. Computer Optics, 2015; 39(1): 70-76. DOI: 10.18287/0134-2452-2015-39-1-70-76.

13. Skidanov RV, Morozov AA. A hyperspectrometer based on diffraction grating with variable height of strokes. Russian Foundation for Basic Research Journal, 2015; 4(88): 89-95. [in Russian]

14. Skidanov RV, Morozov AA, Porfirev AP, Blank VA. An imaging spectrometer based on a discrete interference tilter. Computer Optics, 2015; 39(5): 716-720. [in Russian] DOI: 10.18287/0134-2452-2015-39-5-716-720.

15. Ehrenhaft F. Die Photophorese. Annals of Physics, 1918; 56: 81-132.

16. Porfirev AP, Skidanov RV. Optical trapping and manipulation of light-absorbing particles by means of a Hermite-Gaussian laser beam. Journal of Optical Technology, 2015; 82(9): 587-591.

17. Reicherter M, Haist T, Wagemann EU, Tiziani HJ. Optical particle trapping with computer-generated holograms written on a liquid-crystal display. Optics Letters, 1999; 24: 608610.

18. Khonina SN, Balalayev SA, Skidanov RV, Kotlyar VV, Päivänranta B, Turunen J. Encoded binary diffractive element to form hyper-geometric laser beams. Journal of Optics A: Pure and Applied Optics, 2009; 11(6): 065702.

19. Cohn RW, Liang M. Approximating fully complex spatial modulation with pseudorandom phase-only modulation. Applied Optics, 1994; 33: 4406-4415.

20. Kotlyar VV, Khonina SN, Soifer VA. Methods of encoding composite DOE. Computer Optics, 2001; 21: 36-39. [in Russian]

21. Porfirev AP, Skidanov RV. Dark-hollow optical beams with a controllable shape for optical trapping in air. Optics Express, 2015; 23(7): 8373-8382.

22. Porfirev AP, Skidanov RV. Dynamic photophoresis-based optical trapping using a spatial light modulator. Proceedings of SPIE, 2016; 9884: 98843E. 